University of Wollongong

Research Online

Faculty of Informatics - Papers (Archive)

Faculty of Engineering and Information

Sciences

$1-10-2005$

\title{
Performance investigation on three-classes of MANET routing protocols
}

Mehran Abolhasan

University of Wollongong, mehran.abolhasan@uts.edu.au

Tadeusz A. Wysocki

University of Wollongong,wysocki@uow.edu.au

Justin Lipman

University of Wollongong, jlipman@uow.edu.au

Follow this and additional works at: https://ro.uow.edu.au/infopapers

Part of the Physical Sciences and Mathematics Commons

\section{Recommended Citation}

Abolhasan, Mehran; Wysocki, Tadeusz A.; and Lipman, Justin: Performance investigation on three-classes of MANET routing protocols 2005.

https://ro.uow.edu.au/infopapers/34

Research Online is the open access institutional repository for the University of Wollongong. For further information contact the UOW Library: research-pubs@uow.edu.au 


\title{
Performance investigation on three-classes of MANET routing protocols
}

\author{
Abstract \\ Routing in Ad-hoc Networks has received significant attention with a number of different routing \\ protocols proposed in recent years. These routing protocols may be classified into three main categories: \\ proactive, reactive and hybrid. Prior work aimed at comparing the performance of routing protocols has \\ mainly fo cused on comparing reactive and proactive protocols [6] [4] [1]. In this paper, we present a \\ simulation study of different routing protocols from all three categories. We also explore the benefits and \\ performance of each routing category. Further, we present a discussion of future research directions for \\ routing in Ad-hoc Networks. \\ Disciplines \\ Physical Sciences and Mathematics

\section{Publication Details} \\ This article was originally published as: Abolhasan, $\mathrm{M}$, Wysocki, T and Lipman, J, Performance \\ investigation on three-classes of MANET routing protocols, 2005 Asia-Pacific Conference on \\ Communications, 3-5 October 2005, 774-778. Copyright IEEE 2005.
}




\title{
Performance Investigation on three-classes of MANET Routing Protocols
}

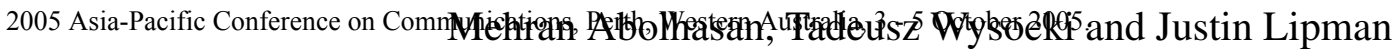 \\ Telecommunications and IT Research Institute (TITR) \\ University of Wollongong, NSW 2522, Australia \\ E-mail: mehran@titr.uow.edu.au
}

\begin{abstract}
Routing in Ad hoc Networks has received significant attention with a number of different routing protocols proposed in recent years. These routing protocols may be classified into three main categories: proactive, reactive and hybrid. Prior work aimed at comparing the performance of routing protocols has mainly focused on comparing reactive and proactive protocols [6] [4] [1]. In this paper, we present a simulation study of different routing protocols from all three categories. We also explore the benefits and performance of each routing category. Further, we present a discussion of future research directions for routing in Ad hoc Networks.
\end{abstract}

\section{INTRODUCTION}

Multi-hop ad hoc networks do not require a fixed infrastructure or a base station and they are made up of a number of enduser nodes. Each node in these networks has the capability to transfer, receive and determine routes other nodes in the network. Moreover, such network can consist of nodes which are static and/or mobile. The ad hoc networks with mobile nodes are commonly referred to as MANETs (or Mobile Ad hoc Networks). The transmission of such networks is usually limited to few hundred meters for mobile devices and can be increased to several KM by using high gain antennas. The limitation in transmission range in such network may often require nodes to establish communications over multiple numbers of hops. Ad hoc networks can provide a cost-effective and easy to configure and maintain networking solution in areas where previous networking infrastructure does not exists. Moreover, such networks can be used to extend the coverage of infrastructured network. The application of such networks spans across various different fields and services. Some of these include: Military communications, Emergency Search and Rescue, extension or provision of Internet type services, development of temporary networks for different events and sensor networking.

Ad hoc networks are still in the early development stage with many research issues still remaining un-resolved. One area of research, which has been a focal point of research in Ad hoc networks is Routing. Routing has received tremendous amount of attention, which has resulted in the proposition of wide range of routing protocols. Generally, Ad hoc routing protocols can be classified into three categories, these are proactive, Reactive and Hybrid.

Proactive (also known as Table-Driven) routing protocols are based on the traditional Link State and Distance Vector algorithms, which were originally designed for wired networks. These protocols maintained and periodically updated their routing tables by exchanging globally propagating control mes-

\footnotetext{
${ }^{1}$ This work is sponsored by Desert Knowledge CRC (DK-CRC).
}

sages. In order to minimise the number of globally propagated routing packets, Localised updating strategy was introduced in protocols such GSR[7]FSR[10]. GSR reduces the number of control overheads transmitted into the network by allowing each node to exchange routing information with their neighbours only. FSR is a descendant of GSR, which further reduced the amount of control overhead by exchanging topology information about nearby nodes at a higher frequency than the more remote ones, using the idea of a fisheye scope [10]. Mobility based updates were introduced in [5] in an effort to eliminate periodic route updates. This was achieved by making the rate at which the route updates occur proportional to the speed at which each node travels. The advantage of this is that in networks with low mobility this updating strategy may produce lower overhead than the periodic routing update approach. A more recent updating strategy first introduced in [9] is known as conditional (or event-based). With this method, updates are sent only if a certain event occurs. For example when a link becomes invalid or when a new node joins the network.

On-demand (or reactive) routing protocols [14][8][15] were introduced to reduce the routing overhead associated with proactive protocols by determining routes when they are required by the source. This is usually done through a two-step process known as Route Discovery. During Route Discovery, when a source node requires a route to a particular destination, a Route Request (RREQ) packet is generated and disseminated throughout the network. If a RREQ packet reaches a node with routing information about the destination or if the destination is found a Route Reply (RREP) is sent back to the source. The source selects its route to the destination based on the route selection criteria employed.

Hybrid routing protocols combine both reactive and proactive routing to increase the overall scalability of routing in $\mathrm{Ad}$ hoc networks. These protocols include: [11][13][19][2]. In these protocols, the network is made up of a number of zones. The network with in each zone is maintained proactively and the routes between zones are determined reactively. The idea behind these strategies is to reduce the number of globally propagating control packets by increasing the level of collaboration between nodes within specific or local regions.

To date, few simulation or test-bed studies have been performed to investigate the performance of a wide range of $\mathrm{Ad}$ hoc network routing protocols [6][4][1]. Furthermore, the majority of the studies performed do not provide a head-to-head comparison of routing strategies based on all three categories of routing protocols. In this paper, we compare all three categories of routing protocols under different networking conditions by using a network simulation package. Furthermore, we 
describe future research required in Ad hoc routing.

The rest of this paper is organised as follows. Section II describes the simulated routing protocols. Section III describes the simulation tool and parameters used. Section IV presents the results of our simulations. Section V presents a discussion in future research directions for routing in Ad hoc networks and Section VI presents the conclusions.

\section{Simulated Protocols}

To compare the performance of the three routing categories, three different routing protocols were chosen. These were OLSR[12], AODV[8] and DZTR[2]. This section describes the functionality of these routing strategies.

\section{A. Optimised Link State Routing (OLSR)}

OLSR [12] is a point-to-point routing protocol based on the traditional link-state algorithm. In this strategy, the nodes maintain topology information about the network by periodically exchanging link-state messages. OLSR attempts to minimise the size of each control message and the number of rebroadcasting nodes during each route update by employing MultiPoint Replaying (MPR) strategy. To do this, during each topology update, each node in the network selects a set of neighbouring nodes to retransmit its packets. This set of nodes is called the multipoint relays of that node. Any node which is not in the set can read and process each packet but do not retransmit. To select the MPRs, each node periodically broadcasts a list of its one hop neighbours using hello messages. From the list of nodes in the hello messages, each node selects a subset of one hop neighbours, which covers all of its two hop neighbours.

\section{B. Ad hoc On-demand Distance Vector (AODV)}

AODV in an on-demand hop-by-hop based routing protocol, which attempts to determine route when it is required by the source. In AODV, when a node has data to send, it checks first to see if it has a valid route to the destination. If a route exists, it uses the known route to send the data to the required destination. Otherwise it initiates the route recovery process. In route discovery, the source node broadcasts a route request to its neighbours. The neighbours also broadcast this route request through their outgoing links to their neighbours. This process continues until a route request reaches the destination node or an intermediate node that has a route to the destination. Each node maintains a sequence number, broadcast ID (which is incremented for every route request generated). The sequence number is used to determine the freshest route to a destination and the broadcast ID is used with the node IP address to generate a unique route request. The sequence number, broadcast ID and the most recent sequence number of the destination are appended to the route request packets. The intermediate nodes with an address to the required destination can only send a route reply if the sequence number associated with the required route is greater or equal to the sequence number in the route request. Each node that forwards the route request creates a reverse route for itself back to the source node. When the destination or a node with the required destination is reached, a route reply is generated which contains the number of hops required to reach the destination and the most recent sequence number seen by the node generating the reply [8]. In AODV routes are maintained by exchanging hello messages with intermediate nodes ${ }^{2}$. For example, a node that does not receive three consecutive hello messages from one of its neighbours, will assume that it no longer has a connection with that particular neighbour. It will then send a route reply with an infinite metric to its upstream neighbours to inform them of the broken link [8].

\section{Dynamic Zone Topology Routing protocol (DZTR)}

The DZTR is a hybrid routing protocol, which is made up of three parts. These are Zone Creation, Topology Determination and Location Discovery. The zone creation phase combines the nodes into dynamic zone. Two zone creation algorithms are proposed in DZTR[3]. These are DZTR1 and DZTR2. In DZTR1, a dynamic zone is created by two non-zone-member nodes. Each zone is identified by zone ID which determines the geographical location of the zone and its boundary. DZTR2, is an extension of DZTR1, which proposes a strategy to reduce partitioning within each zone. The idea behind the creation of dynamic zones is to provide a collaborative platform for nodes in order to minimise the number of redundant control packets.

The topology determination phase is initiated by each node when they become part of a zone. This is used to determine and build the intrazone and interzone topology tables for each node. The intrazone topology table is built and maintained proactively. For example, when a new node comes online or when a node within each zone experiences a significant change in its topology or when the Intrazone Update Timer is expired. The Interzone topology table is built and updated reactively and passively. For example, when a gateway node within each zone detects the presence of a new neighbouring zone through another gateway node or when a link to a neighbouring zone is broken, an interzone update packet is transmitted by the gateway node through its intrazone. The Interzone topology is used to develop a map of the surrounding nodes and how they can be reached.

The location discovery phase of DZTR is initiated when a source node requires a route to a destination. DZTR introduces a number of different strategies to determine a route to a destination with minimal amount of overhead. To show how these strategies work, assume that a node A want to determine a route to a node $\mathrm{D}$. The Node A will begin by performing a query to its routing tables. If an un-expired route is present, then it allows data flow to begin. If the un-expired route is not found, the node A may initiate a number of different location tracking strategies to determine a route to the destination. The location tracking strategy chosen depends on the type of information known about the destination or if the source does not have any information at all [3].

\section{Simulation Model}

This section describes the simulation tool and parameters chosen to simulate the routing protocols. The performance met-

\footnotetext{
${ }^{2}$ Only nodes which are part of an active routes will disseminate hello messages
} 
rics used to compare the performance of the routing protocols are also described.

\section{A. Simulation Environment and Scenarios}

The GloMoSim[16] simulation package was chosen to run the simulations. GloMoSim is an event driven simulation tool designed to carry out large simulations for mobile ad hoc networks. The simulated networks consisted of either 100 or 200 node network, which represented a medium to highly dense ad hoc network. The network was bound to a $1000 \mathrm{~m} \times 1000 \mathrm{~m}$ area. IEEE 802.11 DSSS (Direct Sequence Spread Spectrum) was used with maximum transmission power of $15 \mathrm{dbm}$ at a $2 \mathrm{Mb} / \mathrm{s}$ data rate. In the MAC layer, IEEE 802.11 was used in DCF mode. The radio capture effects were also taken into account. Two-ray path loss characteristic was considered as the propagation model. The antenna height was set to $1.5 \mathrm{~m}$, the radio receiver threshold was set to $-81 \mathrm{dbm}$ and the receiver sensitivity was set to $-91 \mathrm{dbm}$ according to the Lucent wavelan card[17]. Random way-point mobility model was used with the node mobility ranging from 0 to $20 \mathrm{~m} / \mathrm{s}$ and pause time was set to 0 seconds for continuous mobility. The simulations ran for $200 \mathrm{~s}$

and each simulation was averaged over four different simulation runs with different seed values.

Constant Bit Rate (CBR) traffic was used to establish communication between nodes. Each CBR packet contained 64 Bytes and packets were transmitted at 0.25 second intervals. The simulation ran for 10 and 50 different traffic flows and each session began at a randomly selected time and was set to last for the duration of the simulation.

\section{B. Performance Metrics}

The performance of each routing protocol is compared using the following performance metrics.

- Packet Delivery Ratio (PDR)

- Normalised Control Overhead $(\mathrm{O} / \mathrm{H})$

- End-to-End Delay

PDR is the Ratio of the number of packets received by the destination to the number of packets sent by the source. Normalised Control Overhead $(\mathrm{O} / \mathrm{H})$ presents the ratio of the number of control packets transmitted through the network to the number of data packets successfully transmitted. The End-toEnd Delay represents the average delay experienced by each packet when travelling from the source to the destination.

\section{Results}

This section presents the results of our simulation study.

\section{A. Packet Delivery Ratio (PDR)}

Figures 1 and 2 illustrate the PDR achieved for the 100 and 200 node scenarios. In the 100 node scenario both AODV and DZTR produce over 95\% PDR for all different levels of node mobility. OLSR achieved the lowest PDR, ranging from $80 \%$ PDR for constant mobility (i.e. zero pause time) to $94 \%$ for no mobility. OLSR low PDR during high mobility is due to two main reasons. Firstly, it produces significantly more overheads

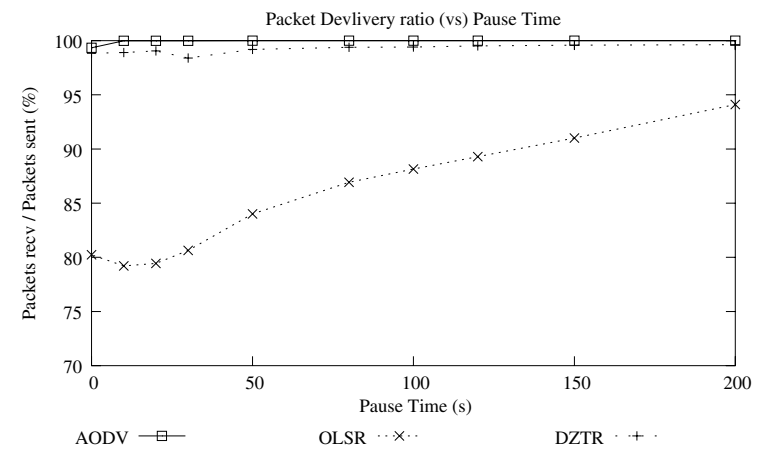

Fig. 1. PDR for $100 \mathrm{~N}$ and 10 Flows

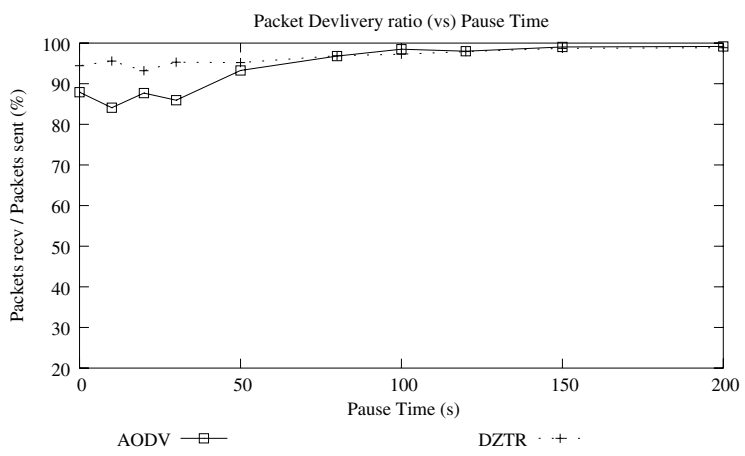

Fig. 2. PDR for $200 \mathrm{~N}$ and 50 Flows

due to the periodic route updates, which increase channel contention and queueing at each node, and results in packets being dropped due to buffer over-flows. Secondly, when mobility is high, periodic route updates may occur infrequently. Therefore, data packets may be dropped due to queueing. The 200 node scenario is used to further investigate the scalability of AODV and DZTR. Here it can be seen that DZTR performs better than AODV when mobility is high. This is because, DZTR significantly reduces the number of control packets transmitted through the network by utilising a number of different location tracking strategies. Therefore, fewer data packets are dropped as a result of channel contention and queueing.

\section{B. Normalised Control Overhead}

Figures 3 and 4 illustrate the normalised overhead introduced into the network. In the 100 node scenario, OLSR produces 6 control packets per data packet transmitted for all levels of pause time. This is 3 times more than AODV, which produces 2 control packets per data packet, when pause time is zero. DZTR produces the lowest amount of control packets when compared to other strategies. This is more evident during zero pause time, where DZTR produces less than 0.3 control packets per data packet, which is over 6 times less than AODV. OLSR's high control overhead is due to the periodical maintenance of twohop neighbour information and period multi-point relay updates in the entire network. In the 200 node scenario, DZTR continues to outperform AODV. The difference between these protocols is again very clear during low pause times, where DZTR produces up to 8 times less control packets than AODV. In both the 100 and 200 node scenarios, the performance difference between AODV and DZTR become more closely matched when 


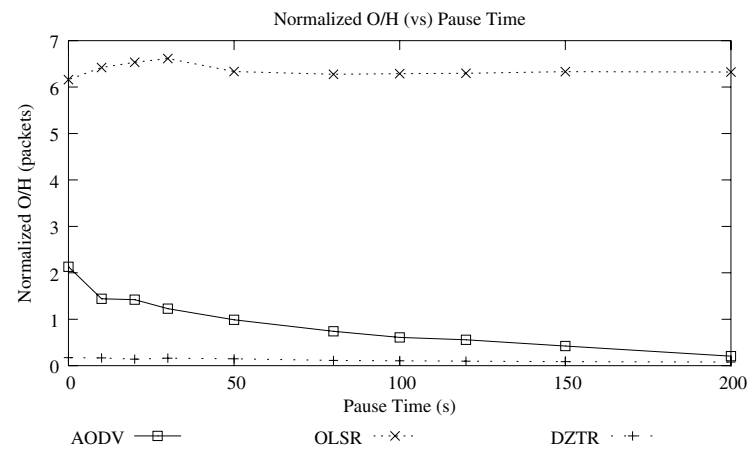

Fig. 3. Normalised $\mathrm{O} / \mathrm{H}$ for $100 \mathrm{~N}$ and 10 Flows

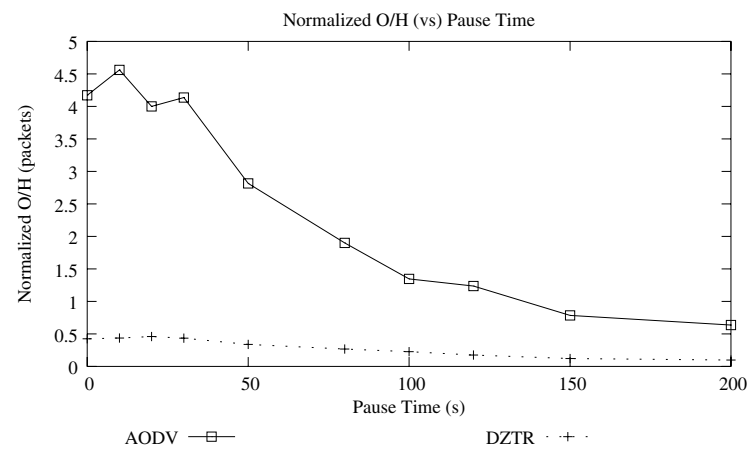

Fig. 4. Normalised $\mathrm{O} / \mathrm{H}$ for $200 \mathrm{~N}$ and 50Flows

pause time is high (i.e. the network becomes more static). This is because, few route re-constructions are required due to route failures caused by broken links.

\section{Delays}

Figures 5 and 6 illustrate the delays produces by each routing protocol. In the 100 node scenario, OLSR produces the lowest end-to-end delay when compared to DZTR and AODV. This is due to the proactive nature of OLSR in which routes are predetermined. Therefore, there may be no initial delays due to un-availability of a route to the destination. However, in AODV and DZTR, routes may need to be determined reactively, which may add extra delay before each data packet can be forwarded to the required destination. DZTR, produces the highest delay, which is on average $15 \mathrm{~ms}$ more than for AODV. This is because, DZTR attempts to find routes using a number of location tracking strategies, which are called one after another if the previous one is not successful. This means that DZTR may go through a number of iteration of route discovery phases before a destination is found. In the 200 node, DTZR produces lower levels of end-to-end delay when compared to AODV. This is due to a higher node density, which increases the probability of a route being found during the first iteration of route discovery in DZTR. Furthermore, with DZTR, the channel contention may also be lower than with AODV, which means that each data packet may spend less time in queues before they are transmitted.

\section{Future RESEARCH}

In the Current Ad hoc network literature, significant amount of research has been devoted to increasing the scalability of $\mathrm{Ad}$

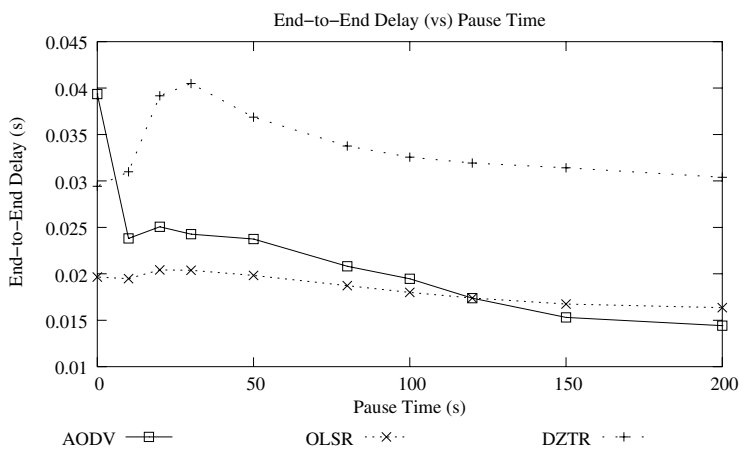

Fig. 5. Delays for $100 \mathrm{~N}$ and 10 Flows

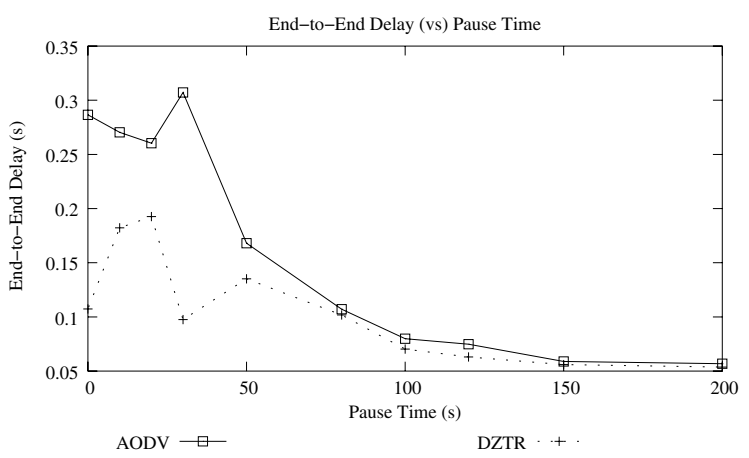

Fig. 6. Delays for $200 \mathrm{~N}$ and 50Flows

hoc routing protocols by introducing different routing structure and hierarchy[13][3][19]. Furthermore, significant amount of research has been performed in on-demand routing to select stable routes and take advantage of prior knowledge about a required destination [18][15][4]. However, little research has been done in increasing scalability when source nodes do not have prior knowledge of destinations. New location tracking strategies and flooding optimisations are required to improve the performance of on-demand routing protocols when node density is high.

\section{CONCLUSIONS}

In this paper we compared three different categories of routing protocols using a simulation study. The protocols simulated where OLSR, AODV and DZTR. From our results, it can be seen that AODV and DZTR are more scalable than OLSR. This is because OLSR relies on two-hop neighbour knowledge information which is determined periodically and introduces significant amount of overhead and channel contention to the network. Furthermore, OLSR uses MPR nodes to re-broadcast route update packets through the network. The disadvantage of this is that MPR nodes may become saturated when node and traffic density is high. Hence, data packets may be dropped due to buffer overflows. DZTR achieves the highest levels of scalability when compared to the other routing strategies. This is due to significantly lower levels of control overheads compared to the other strategies. AODV performs closely to DZTR when node mobility is low. However, when mobility is high DZTR is clearly the more scalable protocol. In the future, we plan to investigate the performance of on-demand and Hybrid Ad hoc 
routing protocols in large networks with high to very high levels

of mobility.

\section{REFERENCES}

[1] M. Abolhasan, T. Wysocki, and E. Dutkiewicz. A Scalability Study of Mobile Ad Hoc Networks Routing Protocols. In Proceedings of sixth International DSPCS, NSW, Australia, 2001.

[2] M. Abolhasan, T. Wysocki, and E. Dutkiewicz. Scalable Routing Strategy for Dynamic Zone-based MANETs. In Proceedings of IEEE GLOBECOM, Taipei, Taiwan, November 17-21 2002.

[3] Mehran Abolhasan, Tadeusz Wysocki, and Eryk Dutkiewicz. Zone-based routing algorithm for mobile ad hoc networks. In Proc. of 1st International Conference on AD-HOC NetwOrks and Wireless (ADHOC-NOW), FIELDS INSTITUTE (Toronto), pages 49-63, September 20-21 2002.

[4] Mehran Abolhasan, Tadeusz Wysocki, and Eryk Dutkiewicz. LPAR: An Adaptive Routing Strategy for MANETs. In Journal of Telecommunication and Information Technology, pages 28-37, 2/2003.

[5] S. Basagni, I. Chlamtac, V.R. Syrotivk, and B.A. Woodward. A Distance Effect Algorithm for Mobility (DREAM). In Proceedings of the Fourth Annual ACM/IEEE International Conference on Mobile Computing and Networking (Mobicom'98), Dallas, TX, 1998.

[6] J. Broch, D. A. Maltz, D. B. Johnson, Y-Ch Hu, and J. Jetcheva. A Performance Comparison of Multi-Hop Wireless Ad Hoc Network Routing Protocols. In Mobile Computing and Networking, pages 85-97, 1998.

[7] T-W. Chen and M. Gerla. Global State Routing: A New Routing Scheme for Ad-hoc Wireless Networks. Proc. IEEE ICC, 1998.

[8] S. Das, C. Perkins, and E. Royer. Ad Hoc On Demand Distance Vector (AODV) Routing. In Internet Draft, draft-ietf-manet-aodv-11.txt, work in progress, 2002.

[9] J.J. Garcia-Luna-Aceves and Cruz Marcelo Spohn. Source-Tree Routing in Wireless Networks. In Proceedings of the Seventh Annual International Conference on Network Protocols Toronto, Canada, page 273, Oct 1999.

[10] M. Gerla. Fisheye State Routing Protocol (FSR) for Ad Hoc Networks. In Internet Draft, draft-ietf-manet-aodv-03.txt, work in progress, 2002.

[11] Z.J. Hass and R Pearlman. Zone Routing Protocol for Ad-Hoc Networks. In Internet Draft, draft-ietf-manet-zrp-02.txt, work in progress, 1999.

[12] P. Jacquet, P. Muhlethaler, T. Clausen, A. Laouiti, A. Qayyum, and L. Viennot. Optimized link state routing protocol for ad hoc networks, ieee inmic pakistan. 2001.

[13] Mario Joa-Ng and I-T Lu. A Peer-to-Peer Zone-based Two-level Link State Routing for Mobile Ad Hoc Networks. IEEE Journal on Selected Areas in Communications, 17(8), 1999.

[14] D. Johnson, D. Maltz, and J. Jetcheva. The Dynamic Source Routing Protocol for Mobile Ad Hoc Networks. In Internet Draft, draft-ietf-manetdsr-07.txt, work in progress, 2002.

[15] Yong-Bae Ko and Nitin H. Vaidya. Location-Aided Routing (LAR) in Mobile Ad Hoc Networks. In Proceedings of the Fourth Annual ACM/IEEE International Conference on Mobile Computing and Networking (Mobicom'98), Dallas, TX, 1998.

[16] UCLA Parallel Computing Laboratory and Wireless Adaptive Mobility Laboratory. Glomosim scalable simulation environment for wireless and wired network systems. In http://pcl.cs.ucla.edu/projects/glomosim/, 2003.

[17] Lucent. Orinoco pc card. In http://www.lucent.com/orinoco, 2003.

[18] C. Toh. A novel distributed routing protocol to support ad-hoc mobile computing. In IEEE 15th Annual International Pheonix Conf. pp 480-86, 1996.

[19] Seung-Chul Woo and Suresh Singh. Scalable Routing Protocol for Ad Hoc Networks. accepted for publication in Journal of Wireless Networks (WINET), 2001. 